Revista de Matemática: Teoría y Aplicaciones 1999 6(2) : 153-174

CIMPA - UCR - CCSS ISSN: 1409-2433

\title{
OPTIMIZA - UN PAQUETE COMPUTACIONAL PARA LA OPTIMIZACIÓN DE PROBLEMAS NO LINEALES
}

\author{
Gonzalo Palencia F.*-Vicente Molina P.* - Venzyslav Llano R.*
}

Recibido: 5 agosto 1998

\begin{abstract}
Resumen
El objetivo del trabajo es construir un software capaz de solucionar problemas de optimización no lineal. Se centra una especial atención en la selección del algoritmo que utiliza basado en el método de Lagrangiano Aumentado combinado con métodos quasi-Newton ( $B F G S, L-B F G S)$. Se explica como está estructurado internamente el software, los pasos para su construcción, la forma de usarlo y se analizan los resultados de las pruebas numéricas realizadas. El sotware OPTIMIZA 3.0 se ejecuta sobre una plataforma Windows (realizado en Borland Delphi 3.0), sus capacidades en cuanto a cantidad de variables y restricciones solo están limitadas por la capacidad de memoria de la máquina utilizada.
\end{abstract}

Palabras Clave: Optimización no lineal, optimización con restricciones, programación matemática, programación no lineal, optimización.

\begin{abstract}
Our goal is to build a software able to solve problems in non linear optimization. A central point is selection of the algorithm, which is based on Augmented Lagrangian Method combined with quasi-Newton methods (BFGS, L-BFGS). The article explains how software is structured, the steps of its construction and the mode of use; furthermore, results and numerical tests are analyzed. OPTIMIZA 3.0 was built on Borland Delphi 3.0 and runs on Windows, its capacity on the number of variables and constraints are only limited on the memory machine to be used.
\end{abstract}

Keywords: Nonlinear optimization, constrained optimization, mathematical programming, nonlinear programming, optimization.

AMS Subject Classification: 90C30, 68-04

* Universidad Central "Marta Abreu" de Las Villas, Facultad de Matemática, Física y Computación, Departamento de Matemática, Carretera Camajuaní Km 5.5, CP - 54830 Santa Clara, Villa Clara, Cuba; E-Mail: gonzalo@udv.etecsa.cu 


\section{Introducción}

Actualmente está bien determinada la importancia de la optimización como uno de los principios básicos del análisis de los problemas complejos de decisión, que implican la selección de valores para cierto número de variables interrelacionadas, centrando la atención en un objetivo diseñado para cuantificar el rendimiento y medir la calidad de la decisión. Este objetivo se maximiza, o minimiza teniendo en cuenta las restricciones que pueden limitar la selección de los valores de las variables de decisión.

Una primera etapa es la elaboración del modelo matemático, donde se realiza el planteamiento del problema de optimización correspondiente. La segunda etapa se refiere a la selección y aplicación de los métodos, algoritmos y herramientas de cálculo necesarios para la búsqueda de la solución del problema matemático y en la tercera etapa se realiza la interpretación y validación práctica de los resultados obtenidos. La solución de los problemas, en la segunda etapa, es el contenido fundamental de la Programación Matemática. Generalmente, en los softwares de optimización no lineal, para lograr efectividad en clases más amplias de funciones se combinan varios algoritmos.

OPTIMIZA es un programa escrito en Borland Delphi v. 3.0 para resolver problemas de optimización del tipo

$$
f(x) \rightarrow \min (\max )
$$

sujeto a:

$$
\begin{aligned}
& h_{i}(x)=0, \quad i=1,2, \ldots, m \\
& g_{j}(x) \leq 0, \quad j=1,2, \ldots, p
\end{aligned}
$$

donde $x \in S \subset \mathbb{R}^{n} ; f: \mathbb{R}^{n} \rightarrow \mathbb{R} ; h: \mathbb{R}^{n} \rightarrow \mathbb{R}^{m} ; g: \mathbb{R}^{n} \rightarrow \mathbb{R}^{p}$, basado en los métodos de Lagrangiano Aumentado [1] para transformar el problema original en otro sin restricciones aplicando entonces a éste Métodos quasi-Newton [1], [2] para obtener una solución. Para obtener una aproximación a la inversa del Hessiano de la función, se utilizan algoritmos del tipo $L-B F G S[3]$.

En la siguiente sección, se muestran los métodos utilizados en OPTIMIZA. En la sección 3 se presenta la estructura general del software. En la sección 4 se describe como utilizarlo y en la sección 5 se relacionan algunos de los experimentos numéricos realizados y se comparan los resultados obtenidos con la solución de los problemas prueba del Generador Schittkowski [4].

\section{El método}

Los problemas generales de la programación no lineal (1) poseen una alta complejidad para solucionarlos. Es por ello que se busca transformarlos en problemas similares que tengan la misma solución, pero de complejidad menor.

\subsection{Conversión del problema a uno sin restricciones}

En la bibliografía el tipo de conversión que más se trata son los métodos de Penalización, donde se propone que se transforme el problema original (1) por otro sin restricciones, 
donde a la función objetivo se le adiciona un término que prescribe un alto costo por la violación de las restricciones, es decir:

Dado el problema (1), obtener

$$
f(x)+c P(x) \rightarrow \min
$$

donde $c>0$ y $P$ es una función en $\mathbb{R}^{n}$ que satisface:

1. $P$ es continua

2. $\forall x \in \mathbb{R}^{n}, P(x) \geq 0$

3. $P(x)=0$ si, y solo si, $x \in S$

Por ejemplo, si $S$ está definido por cierto número de restricciones de desigualdad

$$
S=\left\{x: g_{j}(x) \leq 0 j=1,2, \ldots, p\right\}
$$

una función de penalización muy útil es la definida por

$$
P(x)=\frac{1}{2} \sum_{j=1}^{p}\left(\max \left[0, g_{j}(x)\right]^{2}\right.
$$

con lo cual se garantizan las condiciones exigidas para $p$ y además su continuidad y diferenciabilidad si las funciones $g_{j}, j=1,2, \ldots, p$ lo son.

Por otra parte, una de las clases generales de métodos de programación no lineal más efectivos es la de los métodos de Lagrangiano Aumentado [1], también llamados Métodos de los Mutiplicadores. Estos métodos se pueden considerar como métodos combinados de funciones de penalización y de dualidad local.

En el método de Lagrangiano Aumentado para resolver problemas del tipo

$$
f(x) \rightarrow \min
$$

$$
\text { sujeto a } h(x)=0
$$

se toma la función $f(x)+\lambda^{t} h(x)+\frac{1}{2} c|h(x)|^{2}$, donde $x \in \mathbb{R}^{n}, c \in \mathbb{R}^{+}, \lambda \in \mathbb{R}^{m}$.

Desde el punto de vista de una función de penalización, el Lagrangiano Aumentado, para un valor fijo del vector $\lambda$, es simplemente la función de penalización cuadrática estándar para el problema

$$
\begin{gathered}
f(x)+\lambda^{t} h(x) \rightarrow \min \\
\text { sujeto a } h(x)=0 .
\end{gathered}
$$

Se observa que el problema (3) es equivalente al problema (2), pues las combinaciones de las restricciones asignadas a $f(x)$ no alteran el punto mínimo ni el valor mínimo.

El Lagrangiano Aumentado se considera como una función de penalización exacta cuando se utiliza el valor adecuado de $\lambda^{*}$ y se demuestra en [1] que una buena aproximación a la incógnita $\lambda^{*}$ es $\lambda_{k+1}=\lambda_{k}+\operatorname{ch}\left(x_{k}\right)$. 
Desde el punto de la teoría de la dualidad, el Lagrangiano Aumentado es simplemente el Lagrangiano estándar para el problema

$$
\begin{gathered}
f(x)+\frac{1}{2} c|h(x)|^{2} \rightarrow \min \\
\text { sujeto a } h(x)=0 .
\end{gathered}
$$

Este problema equivale al problema original (2), pues la suma del término $\frac{1}{2} c|h(x)|^{2}$ al objetivo no cambia el valor optimal, el punto solución óptimo, ni el multiplicador de Lagrange. Sin embargo, mientras que el Lagrangiano original puede no ser convexo cerca de la solución, el término $\frac{1}{2} c|h(x)|^{2}$ tiende a hacer convexo el Lagrangiano. De hecho, para $c$ suficientemente grande el Lagrangiano será localmente convexo.

Como en el problema (2) se tratan solo ecuaciones de igualdad habrá que realizar una pequeña transformación a las restricciones de desigualdad del problema original (1). Sea el problema

$$
\begin{gathered}
f(x) \rightarrow \min \\
\text { sujeto a } g(x) \leq 0,
\end{gathered}
$$

donde $f: \mathbb{R}^{n} \rightarrow \mathbb{R}, g: \mathbb{R}^{n} \rightarrow \mathbb{R}^{p}, x \in \mathbb{R}^{n}$. Se supone que este problema tiene una solución bien definida $x^{*}$, que es un punto regular de las restricciones que satisface las condiciones de suficiencia de segundo orden para un mínimo local. Este problema se puede formular como un problema equivalente con restricciones de igualdad:

$$
f(x) \rightarrow \min
$$

$$
\text { sujeto a } g_{j}(x)+z_{j}^{2}=0, \quad j=1,2, \ldots, p \text {. }
$$

Haciendo un ánalisis de como determinar $z$, en [1] se llega a la siguiente expresión:

$$
z_{j}^{2}= \begin{cases}-g_{j}(x)-\frac{\mu_{j}}{c} & \text { si }-g_{j}(x)-\frac{\mu_{j}}{c}>0 \\ 0 & \text { en el resto de los casos. }\end{cases}
$$

O, de otra forma, $z_{j}^{2}=\max \left[0,-g_{j}(x)-\frac{\mu_{j}}{c}\right]$ quedando el Lagrangiano para el caso de restricciones de desigualdad en la forma

$$
L_{c}=f(x)+\sum_{j=1}^{p} \frac{1}{2} c\left\{\left[\max \left(0, \mu_{j}+c g_{j}(x)\right)\right]^{2}-\mu_{j}^{2}\right\} .
$$

Entonces para el problema (5) el multiplicador de Lagrange $\mu$ se puede ajustar igual que en el caso de igualdad.

\subsection{Solución del problema sin restricciones}

Para resolver el problema sin restricciones, en [2] se hace referencia a diversos algoritmos de optimización no lineal sin restriciones que han sido los más utilizados hasta ese momento. En particular, los métodos quasi-Newton comienzan tomando un punto inicial $x_{0}$ 
y van generando una sucesión de puntos que converjan al mínimo o máximo buscado. La búsqueda se hace de la siguiente manera:

$$
x_{k+1}=x_{k}+\alpha_{k} d_{k}, d_{k}=-H_{k} q_{k}, q_{k}=\nabla L_{c}\left(x_{k}\right)
$$

donde: $H_{k}$ es una aproximación a la inversa del Hessiano de la función, y $\alpha_{k}$ es el resultado de la búsqueda lineal para encontrar el mínimo de la función en la dirección de descenso.

Son varios los métodos para calcular la aproximación a la inversa del Hessiano pero, como se aprecia en [2], donde se hace una comparación entre varios de estos métodos, se tiene a los algoritmos de la familia Broyden, y en especial a los $B F G S$, como los candidatos más favorecidos.

Lo que el algoritmo propone realizar es una actualización de la forma:

$$
H_{k+1}^{B F G S}=V_{k}^{t} H_{k} V_{k}+\rho_{k} s_{k} s_{k}^{t},
$$

donde $s_{k}=x_{k+1}-x_{k}, y_{k}=g_{k+1}-g_{k}, \rho_{k}=\frac{1}{y_{k}^{t} s_{k}}$, y $V_{k}=I-\rho_{k} y_{k} s_{k}^{t}$.

Los métodos quasi-Newton se suelen ejecutar de manera continua, comenzando con una aproximación inicial y mejorándola sucesivamente mediante el proceso iterativo. Bajo ciertas condiciones algo estrictas se puede demostrar que este procedimiento es globalmente convergente [1]. Por otro lado, si los métodos quasi-Newton se inician de nuevo cada $n$ ó $n+1$ pasos volviendo a hacer la aproximación a la inversa del Hessiano igual a su valor inicial, la convergencia global se garantiza mediante la presencia del primer paso descendente de cada ciclo (que actúa como un paso espaciador).

El algoritmo para solucionar el problema sin restricciones nos quedaría de la forma siguiente:

Paso 0. Dado $x_{0}$ y $H_{0}$ matriz definida positiva; $k=0$

Paso 1. Calcular

$$
\begin{aligned}
d_{k} & =-H_{k} q_{k} \\
x_{k+1} & =x_{k}+\alpha_{k} d_{k}
\end{aligned}
$$

donde $\alpha$ satisface las condiciones de Wolfe:

$$
\begin{aligned}
& f\left(x_{k}+\alpha_{k} d_{k}\right) \leq f\left(x_{k}\right)+\beta^{\prime} \alpha_{k} q_{k}^{t} d_{k} \\
& q\left(x_{k}+\alpha_{k} d_{k}\right) \geq \beta q_{k}^{t} d_{k}
\end{aligned}
$$

y se propone comenzar con $\alpha=1 ; 0<\beta^{\prime}<\beta<1$.

Paso 2. Calcular $q_{k+1}=q\left(x_{k+1}\right)$

Paso 3. Verificar, si $\left|q_{k+1}\right|<\varepsilon$ entonces fin, si no calcular $H_{k+1}$ e ir al Paso 1. 
Este método necesita $\mathcal{O}\left(n^{2}\right)$ localizaciones de memoria, mientras que, como se muestra en [3], una variante con las mismas propiedades de convergencia que el anterior, denominado $L-B F G S$, propone tomar la matriz de actualización como:

$$
\begin{aligned}
H_{k+1}^{L-B F G S}= & \left(V_{k}^{t} \ldots V_{k-\widehat{m}}^{t}\right) H_{0}\left(V_{k-\widehat{m}} \cdots V_{k}\right)+ \\
& +\rho_{k-\widehat{m}}\left(V_{k}^{t} \cdots V_{k-\widehat{m}+1}^{t}\right) s_{k-\widehat{m}} s_{k-\widehat{m}}^{t}\left(V_{k-\widehat{m}+1} \cdots V_{k}\right)+ \\
& +\rho_{k-\widehat{m}+1}\left(V_{k}^{t} \cdots V_{k-\widehat{m}+2}^{t}\right) s_{k-\widehat{m}+1} s_{k-\widehat{m}+1}^{t}\left(V_{k-\widehat{m}+2}^{t} \cdots V_{k}\right)+\cdots+\rho_{k} s_{k} s_{k}^{t} .
\end{aligned}
$$

Hay que notar que la matriz $H_{k}$ no se forma explícitamente, sino que los $\widehat{m}+1$ valores de $y_{j}$ y $s_{j}$ son almacenados separadamente y con ellos se realiza la actualización. Lo que se propone es actualizar $m$ pares de datos y hacer la actualización de la matriz $H_{k+1}$ con solo esta información, pero se obtiene una matriz semejante en cuanto a resultados numéricos a la del $B F G S$.

Para seleccionar el valor de $m$, es decir, la cantidad de datos que se almacenarán, se demuestra en [3] que con base en las pruebas numéricas realizadas una buena selección es tomar $m=5$, y que tomar valores mayores no incrementa realmente el rendimiento del algoritmo. El Paso 3 nos quedaría entonces de la forma:

Paso 3. Calcular $\widehat{m}=\min \{k, m-1\}$.

Actualizar $H_{0}$ en $\widehat{m}+1$ veces usando los pares $\left\{y_{j}, s_{j}\right\}_{j}^{k}=k-\widehat{m}$

$H_{k+1}^{L-B F G S}$ se actualiza de acuerdo con (7)

Hay que notar que esta actualización es muy fácil de implementar y es similar en complejidad al BFGS, con la diferencia en el cálculo de la matriz.

\subsection{Búsqueda Lineal}

Ya encontrada la dirección de descenso en la cual la función objetivo decrece, se debe buscar el valor por el cual se debe multiplicar el vector de dirección $d$ para lograr un decrecimiento efectivo. Esta $\alpha$ se pudiera calcular prácticamente exacta, pero habría que pagar un alto costo computacional y la correspondiente pérdida de tiempo, es por esto que se ha desarrollado toda una teoría de como realizar la búsqueda lineal imprecisa, pero que garantice que la función decrezca. Diferentes métodos relacionados con lo anterior se estudian en [1]-[3] y todos coinciden en buscar $\alpha$ que no sea demasiado grande o pequeña, o sea, que debe satisfacer las condiciones de Wolfe (6).

En [4] se propone que se realice esta búsqueda comenzando con $\alpha=1$ y se verifican las desigualdades (6).

El algoritmo para determinar la amplitud del paso nos quedaría de la forma siguiente:

Paso 0. Tomar $\alpha=1, \beta \in(0,1)$

Paso 1. Verificar las condiciones de Wolfe (6)

Paso 2. Si no se cumplen, tomar $\alpha=\frac{1}{2} \alpha$, asignar $\alpha_{k}=\alpha$ e ir al Paso 1, en caso contrario $x_{k+1}=x_{k}+\alpha_{k} d_{k}$ 
En el presente trabajo se utilizan los valores $\beta^{\prime}=10^{-4}$ y $\beta=0.9$ los cuales son recomendados en [2], [5], [6].

\subsection{Escalas para mejorar la convergencia del algoritmo}

Es conocido que una simple variación de la escala de las variables puede provocar una mejora considerable en el comportamiento del algoritmo, por lo que pasaremos a realizar algunas observaciones en este sentido.

En [3] se plantean cuatro tipos de escalas para mejorar la matriz $H_{k}$ y las propuestas son:

- Escala M1: $H_{k}^{(0)}=H_{0}($ no escalada $)$

- Escala M2: $H_{k}^{(0)}=\gamma_{0} H_{0}$ (solo un escalado inicial)

- Escala M3: $H_{k}^{(0)}=\gamma_{k} H_{0}$

- Escala M4: Igual a M3 durante las $m$ primeras iteraciones.

Para $k>m, H_{k}^{(0)}=D_{k}$, donde $\gamma_{k}=\frac{y_{k}^{t} s_{k}}{\left\|y_{k}\right\|^{2}}$ y $D_{k}=\operatorname{diag}\left(d_{k}^{i}\right)$. En el trabajo de referencia se realizaron varios experimentos, obteniéndose los mejores resultados con la escala M3.

\section{Algoritmo Final de Solución}

Dados $f, g, h$

Paso 0. Inicializar $\lambda, \mu, c, H_{0}, x_{0}$

Paso 1. Calcular $L_{c}, q_{k}=\nabla L_{c}\left(x_{k}\right)$

Paso 2. Si $\left|q_{k}\right|<\varepsilon$, entonces

$$
\left\{\begin{array}{l}
\text { Si }|h(x)|<\varepsilon, \text { entonces }\left\{\begin{array}{l}
\text { si } g(x) \leq \varepsilon, \text { fin } \\
\text { si } g(x)>\varepsilon, \text { actualizar } \mu
\end{array}\right. \\
\text { en caso contrario, actualizar } \lambda \text { y si } g(x)>\varepsilon, \text { actualizar } \mu
\end{array}\right.
$$

Actualizar $L_{c}$

Paso 3. Calcular un nuevo punto

$$
d_{k}=-H_{k} q_{k}, x_{k+1}=x_{k}+\alpha_{k} d_{k}
$$

Hallar $q_{k+1}$ e ir al Paso 2 . 


\section{Estructura de OPTIMIZA}

\subsection{El compilador}

\section{Unit Scanner}

En esta unit se define la función Scan, a la cual se le pasa como parámetro una cadena de caracteres que es dividida en tokens de acuerdo con los símbolos divisores que se especifican en :

Nestid: símbolos que indican anidamiento de estructuras y

Primary: símbolos divisores primarios.

Los tokens son clasificados en varios tipos y devueltos en una lista por la función.

\section{Unit Parser}

Esta unit contiene la clase TParser, la cual exporta un conjunto de métodos que a partir de una lista de tokens verifican que estos se encuentren en un correcto orden sintáctico de acuerdo con las reglas gramaticales definidas y construyen una representación interna del objeto específico que se trate, devolviendo una referencia a dicho objeto. Los métodos son los que se presentan a continuación:



\section{Unit Constants}

Todas las constantes usadas en la interfaz con el usuario son definidas en esta unit. Se definen los identificadores de las funciones matemáticas y los signos de las relaciones algebraicas.

\subsection{El núcleo matemático}

\section{Unit MathObject}

El objeto matemático, TMathObject, es la clase base de todas las que conforman el núcleo matemático. A partir de esta clase se especializan las siguientes:

TNumber

TExpression

TMatrix

TRelation

La clase TNumber encapsula a un número real, a través de la propiedad Value se accede a su valor. 


\section{Unit Struct}

Una de las principales ramas de nuestra jerarquía de clases se encuentra definida en esta unit a partir de TExpression, base de todo el andamiaje matemático de esta aplicación.

Una expresión matemática puede ser una constante (TConstant), o una variable o constante literal (TIdentity) o una concatenación de cualesquiera de ellas enlazadas a través de operaciones matemáticas. Estas operaciones pueden ser binarias (TBinaryOperation) o unarias (TUnaryOperation) dependiendo de la cantidad de argumentos sobre los que actúan.

Toda expresión se puede evaluar a través de la propiedad Value, y mediante los métodos:

function Derivative(Respect: TIdentity): TExpression;

procedure Simplify;

se puede derivar con respecto a una variable y se puede simplificar respectivamente una expresión.

Las operaciones unarias tienen la propiedad Child, que es la expresión sobre la cual se realiza la operación; mientras que las binarias tienen las propiedades LeftChild y RightChild. Estas propiedades son renombradas en las clases descendentes de acuerdo a sus características.

De TUnaryOperation heredan las siguientes clases:

TNeg(X); es el negativo del argumento, $-\mathrm{X}$

$\operatorname{TInv}(\mathrm{X})$; es el inverso del argumento, $\frac{1}{X}$

$\mathrm{TExp}(\mathrm{X})$; es el exponencial de $\mathrm{X}, \mathrm{e}^{x}$

TNeperian(X); es el logaritmo neperiano de X, Ln X

Además heredan también las funciones trigonométricas y sus inversas:

TSin, Tcos, TTan, TCot, TArcSin, TArcCos, TArcTan; y las funciones hiperbólicas y sus inversas: TSinh, TCosh, TTanh, TCoth, TArcSinh, TArcCosh, TArcTanh.

De TBinaryOperation heredan las siguientes clases:

TSum $(\mathrm{X}, \mathrm{Y})$; es la suma de $\mathrm{X}+\mathrm{Y}$

TProd $(\mathrm{X}, \mathrm{Y})$; es el producto de $\mathrm{X} * \mathrm{Y}$

TPower $(X, Y)$; es $X$ elevado a la potencia $Y$.

TRoot $(X, Y)$, es la raíz $Y$-ésima de $X$.

Se define en esta unit además las clases de matrices TPointMatrix y TPointVector, especializadas para manipular variables (TIdentity).

\section{Unit Matrix}

Las propiedades y el comportamiento básico de las matrices son definidas en esta unit a través de la clase TMatrix.

Nuestras matrices pueden contener elementos de cualquier tipo de objeto matemático, pero todos los elementos que contenga una matriz tienen que ser de la misma clase y dicha clase se especificará en la propiedad ElementType. Para describir la cantidad de filas y columnas de una matriz se utlizan las propiedades RowsCount y ColumsCount 
respectivamente y para acceder a un elemento específico se hace a través de la property Elements [const row, col:integer]:TMathObject;

También se define en esta unit TVector, clase que hereda la estructura de TMatrix y especializa su interfaz para hacerla más cercana a los términos matemáticos. Así incorpora una nueva propiedad, VectorType, para describir si se trata de un vector fila o columna.

Una matriz especializada en contener números es TNumericMatrix, que además de tener el mismo comportamiento de su ancestro TMatrix incorpora nuevos métodos para realizar operaciones entre matrices numéricas como la multiplicación, la adición y la multiplicación por un escalar. También se define TNumericVector.

\section{Unit Relations}

En esta unit se define la clase TRelation, que nos permite establecer relaciones de dos tipos. El primero son las funciones explícitas definidas por los usuarios que relacionan a un identificador con una expresión algebraica mediante la clase TUser-Function. El segundo es la relación que se puede establecer entre expresiones: mayor que, menor que, igualdad, etc, a través de la clase TAlgebricRelation.

En la interfaz de TRelation se encuentran las propiedades LeftExpression y RightExpression que son evidentemente las expresiones que se relacionan; el signo que establece la relación se escribe en Sign, y el método Simplify trata de hacer más simple las expresiones relacionadas.

Las principales propiedades de TUserFunction son:

Identifier: es el nombre que identifica a la función.

Expression: es el cuerpo mismo de la función.

Variable [i] : es la variable i-ésima de la función.

VariablesCount: es la cantidad de variables que están presentes en la expresión de la función.

El método

function EvaluateAt(Point: TPointVector): TNumber;

brinda un mecanismo para evaluar la función en un punto arbitrario Point.

La clase TAlgebricRelation se especializa en TEquation y TInequation, es decir, ecuaciones e inecuaciones.

En esta unit se define además TFunctionalMatrix y TFunctionalVector ambas descendientes de TMatrix y especializadas en contener funciones. Estas clases de matrices se pueden evaluar en un punto a través del método

function EvaluateAt(Point:TPointVector): TNumericMatrix; devolviendo una matriz numérica.

\subsection{El problema a resolver}

\section{Unit Problem}

En esta unit se encuentra la clase TProblem la cual se encarga de encapsular el problema de optimización que se trata de resolver y que tiene la forma:

$$
f(x) \rightarrow(\max , \min )
$$




$$
\text { sujeto a } h(x)=0, g(x)<0 .
$$

En su interfaz encontramos las siguientes propiedades:

ObjetiveFn: Es la función que será objeto de la optimización.

SolveObjetive: Indica si el problema se quiere maximizar o minimizar.

Constraint [i]: Es la restricción i-ésima.

ConstraintsCount: Indica la cantidad de restricciones que tiene el problema.

Variable [i]: Permite acceder a la variable i-ésima.

VariablesCount: Es la cantidad de variables que contiene el problema.

Defined [i]: Permite acceder al identificador de la expresión auxiliar i-ésima.

Definition [i]: Es la expresión auxiliar i-ésima

DefinitionsCount: Indica la cantidad de expresiones auxiliares que se han definido en el problema.

Gradient: Es el gradiente de la función objetivo.

Además de estas propiedades TProblem tiene los métodos:

procedure AddConstraint(aConstraint: TAlgebricRelation);

procedure AddDefinition(aDefinition: TUserFunction);

que se utilizan para añadir restricciones y añadir expresiones auxiliares al problema respectivamente.

\subsection{Los métodos de solución}

\section{Unit Methods}

En esta unit se encuentran la clase básica TSolveMethod que encapsulará cualquier algoritmo para la solución del problema a optimizar, a su vez, la clase TLinealSearch encapsula los algoritmos de búsqueda lineal.

TSolveMethod exporta la función Resolve que como parámetro recibe el problema a optimizar y devuelve el punto donde dicho problema es óptimo.

La clase TLBFGSMethod es la implementación del algoritmo de optimización de modelos BFGS de memoria limitada.

\section{El uso de OPTIMIZA}

\subsubsection{Uso de la interfaz gráfica}

La interfaz de usuario que utiliza el paquete Optimiza es un esquema multipágina que es de gran comodidad ya que va guiando paso a paso al usuario en el proceso de entrar los datos de un problema y darle solución, especificando en cada momento la acción que debe realizarse y comprobando que está correcta antes de pasar al próximo paso.

En el borde inferior de la ventana principal se encuentra la barra de estado que brinda una breve orientación sobre la utilización de cada uno de los controles que aparecen en la aplicación. Para una explicación más detallada se puede solicitar el servicio de ayuda presionando la tecla F1 o buscar en el submenú Help o hacer click sobre los botones de ayuda. 
Cuando se comienza la aplicación, la primera página que aparece activa lleva el título General. En esta página aparece un conjunto de atributos del problema que, opcionalmente, se puede ignorar:

- Name: Aquí escribirá el nombre del problema.

- Classification: Hace referencia a alguna clasificación del problema.

- Source: Es la fuente de origen del problema.

- Comments: Cualquier comentario que desee hacer sobre el problema.

Hay también en esta página dos incisos que sí es obligatorio especificar: Variables y Constraints que significan el número de variables y el número de restricciones que respectivamente contiene el problema.

Para editar cualquiera de estos incisos debe hacer click en el panel que aparece a la derecha de la etiqueta que lo identifica.

La próxima página titulada Functions permite la edición de la función que desee optimizar y de funciones auxiliares que podrá utilizar para hacer más cómoda y comprensible la edición de las expresiones que utilice.

Debajo del texto Objetive function aparece un botón que puede tener dos estados min minimizar y max maximizar la función objetivo, para conmutar el estado haga un click sobre él. A la derecha de este botón hay una caja para editar la función objetivo.

Bajo el texto Definitions se puede editar una lista de funciones auxiliares haciendo click sobre los paneles que aparecen al mover el puntero del mouse sobre esa región.

La página siguiente se denomina Constraints y es la que permite editar las restricciones del problema.

La siguiente página Start contiene una lista de las variables del problema, al lado de cada una de ellas se escribirá el valor inicial que tomarán en el momento de aplicar el algoritmo de solución del problema. El valor inicial implícito es cero.

La última página Solution es la que mostrará los resultados de aplicar el algoritmo de solución al problema planteado. Al seleccionar esta página se echa a andar el mecanismo de cálculo y luego se podrán observar los resultados del mismo.

OPTIMIZA brinda también la posibilidad de trabajar con archivos fuente. Es decir, usted puede escribir con ayuda de un editor de texto un archivo en formato texto MS-DOS que contenga las especificaciones necesarias para optimizar el modelo de un problema (vea en el punto 4.3 el formato de los ficheros fuente) y luego el paquete será capaz de leerlo y ejecutarlo.

\subsubsection{Gramática de las expresiones matemáticas}

Las expresiones matemáticas se definen como una constante numérica o una constante literal o una variable o una concatenación de cualesquiera de ellas enlazadas a través de operaciones matemáticas como suma, resta, multiplicación, división, funciones de potencia, funciones trigonométricas y otras.

La tabla siguiente ilustra la sintaxis en la escritura de cada uno de los elementos que constituyen las expresiones matemáticas. 


\begin{tabular}{|c|c|}
\hline Expresión & Sintaxis \\
\hline Constante numérica & $\langle$ número $\rangle$ \\
\hline Constante literal & $\langle$ texto $\rangle$ \\
\hline Variable & $\mathrm{X}\langle$ número $\rangle$ \\
\hline Suma & $\langle$ expresión $\rangle+\langle$ expresión $\rangle$ \\
\hline Producto & $\langle$ expresión $\rangle *\langle$ expresión $\rangle$ \\
\hline Resta & $\langle$ expresión $\rangle-\langle$ expresión $\rangle$ \\
\hline División & $\langle$ expresión $\rangle /\langle$ expresión $\rangle$ \\
\hline Potencia & Power ( $\langle$ expresión $\rangle,\langle$ expresión $\rangle)$ \\
\hline Exponencial & $\operatorname{Exp}(\langle\operatorname{expresión}\rangle)$ \\
\hline Logaritmo neperiano & Ln( (expresión) \\
\hline Seno & Sin (〈expresión $\rangle)$ \\
\hline Coseno & Cos (〈expresión $\rangle)$ \\
\hline Tangente & Tan (〈expresión $\rangle)$ \\
\hline ArcSeno & ArcSin ( $\langle$ expresión $\rangle)$ \\
\hline ArcCoseno & $\operatorname{ArcCos}(\langle$ expresión $\rangle)$ \\
\hline ArcTangente & ArcTan ( $\langle$ expresión $\rangle)$ \\
\hline Seno hiperbólico & Sinh ( $\langle$ expresión $\rangle)$ \\
\hline Coseno hiperbólico & Cosh ( $($ expresión $\rangle)$ \\
\hline Tangente hiperbólica & Tanh ( $\langle$ expresión $\rangle)$ \\
\hline ArcSeno hiperbólico & ArcSinh ( $($ expresión $\rangle)$ \\
\hline ArcCoseno hiperbólico & Arcosh ( $($ expresión $\rangle)$ \\
\hline ArcTang. hiperbólica & ArcTanh ( $\langle$ expresión $\rangle)$ \\
\hline
\end{tabular}



\subsubsection{Formato de los ficheros fuente}

Los ficheros fuente de OPTIMIZA deberán ser escritos siguiendo las siguientes reglas:

- El fichero debe ser un fichero texto del formato MS-DOS.

- No hay diferencias entre las letras mayúsculas y minúsculas.

- Debe comenzar con la palabra PROBLEMA seguida de un texto entre comillas que se tomará como el título.

- Cualquier texto precedido de "//" será ignorado.

- Cualquier texto precedido de “/\$" será tomado como el comentario que se visualiza en la segunda pantalla.

- Antes de hacer referencia a una variable debe aparecer la claúsula VARIABLES seguida del número de variables que usará, no se permite hacer referencias a variables de índice mayor que el especificado.

- Si quiere especificar el significado de una variable lo puede hacer digitando el nombre de la variable seguida de su significado entre comillas. 
- La función objetivo se define como:

Max = expresión matemática ó

Min = expresión matemática.

- La forma de representar las restricciones es:

Expresión matemática $=$ Expresión matemática

Expresión matemática 〈Expresión matemática

Expresión matemática $\rangle$ Expresión matemática NoNeg(variable inicial,variable final).

- Las especificaciones deben terminar con la claúsula FIN y cualquier texto que aparezca después será ignorado.

\section{Experimentos numéricos}

OPTIMIZA fue validado utilizando los ejemplos-prueba que se presentan en el generador Schittkowski [4]. Estos problemas fueron resueltos en una PC 586 a $220 \mathrm{MHz}$ con $32 \mathrm{Mb}$ de memoria RAM y se utilizó una precisión numérica de $10^{-14}$, obteniéndose la solución correcta en 80 problemas. A continuación se muestran los resultados obtenidos durante la ejecución del programa de algunos problemas, comparando al mismo tiempo con las soluciones que brinda el generador de problemas.

\section{Problema 1}






\section{Problema 3}

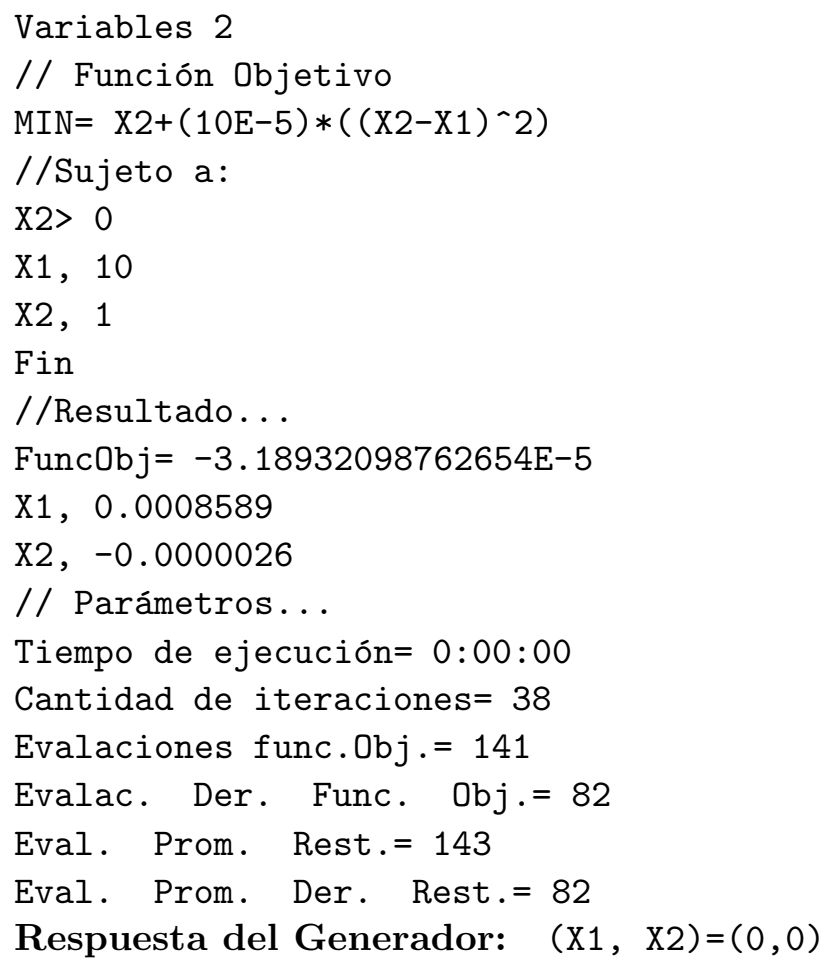

\section{Problema 4}

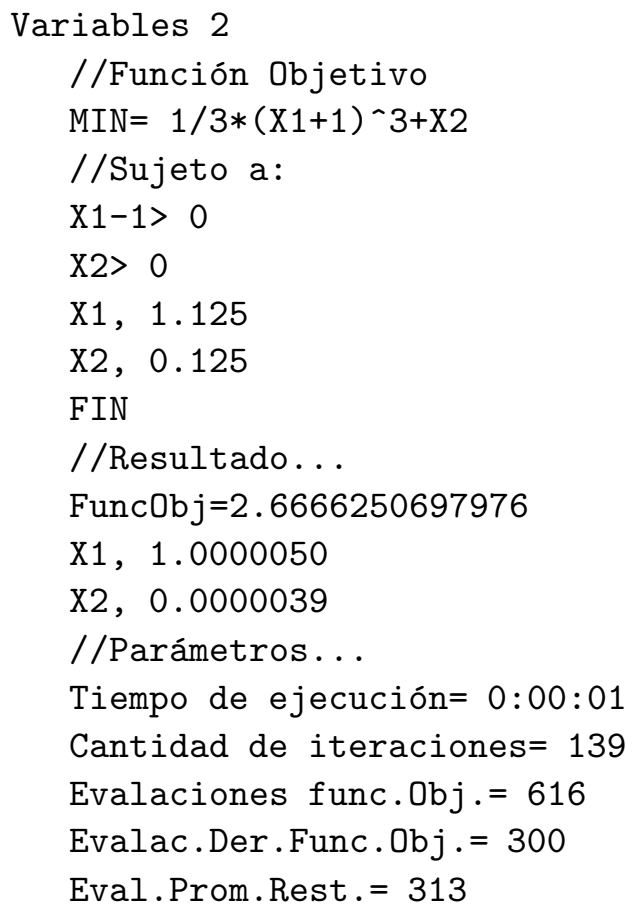


Eval.Prom.Der $\cdot$ Rest $\cdot=150$

Respuesta del Generador: $\quad(\mathrm{X} 1, \mathrm{X} 2)=(1,0)$

\section{Problema 5}

Variables 2

//Función Objetivo

$\mathrm{MIN}=\mathrm{SEN}(\mathrm{X} 1+\mathrm{X} 2)+(\mathrm{X} 1-\mathrm{X} 2) \wedge 2-1.5 * \mathrm{X} 1+2.5 * \mathrm{X} 2+1$

$/ /$ Sujeto a:

$\mathrm{X} 1-4<0$

$\mathrm{X} 1+1.5>0$

$\mathrm{X} 2-3<0$

$\mathrm{X} 2+3>0$

Fin

//Resultado...

FuncObj $=-1.91322258616816$

$\mathrm{X} 1,-0,5470162$

$\mathrm{X} 2,-1.5467840$

//Parámetros...

Tiempo de ejecución= 0:00:00

Cantidad de iteraciones $=28$

Evalaciones func.Obj. $=114$

Evalac. Der. Func.0bj. $=60$

Eval.Prom. Rest. $=28$

Eval. Prom. Der. Rest. $=15$

Respuesta del generador: $(x 1, x 2)=\left(-\frac{\pi}{3}+\frac{1}{2},-\frac{\pi}{3}-\frac{1}{2}\right)$

\section{Problema 6}

Variables 2

//Función Objetivo

$\mathrm{MIN}=(1-\mathrm{X} 1) \wedge 2$

//Sujeto a:

$10 *(\mathrm{X} 2-\mathrm{X} 1 \wedge 2)=0$

$\mathrm{X} 1,-1.2$

$\mathrm{X} 2,1$

Fin

//Resultado...

FuncObj= $1.82602346861371 \mathrm{E}-12$

$\mathrm{X} 1,0.9999990$

$\mathrm{X} 2,0.9999979$

//Prámetros...

Tiempo de ejecución= 0:00:01

Cantidad de iteraciones $=141$ 


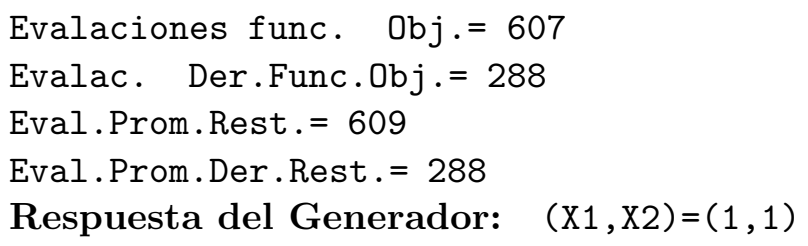

\section{Problema 31}

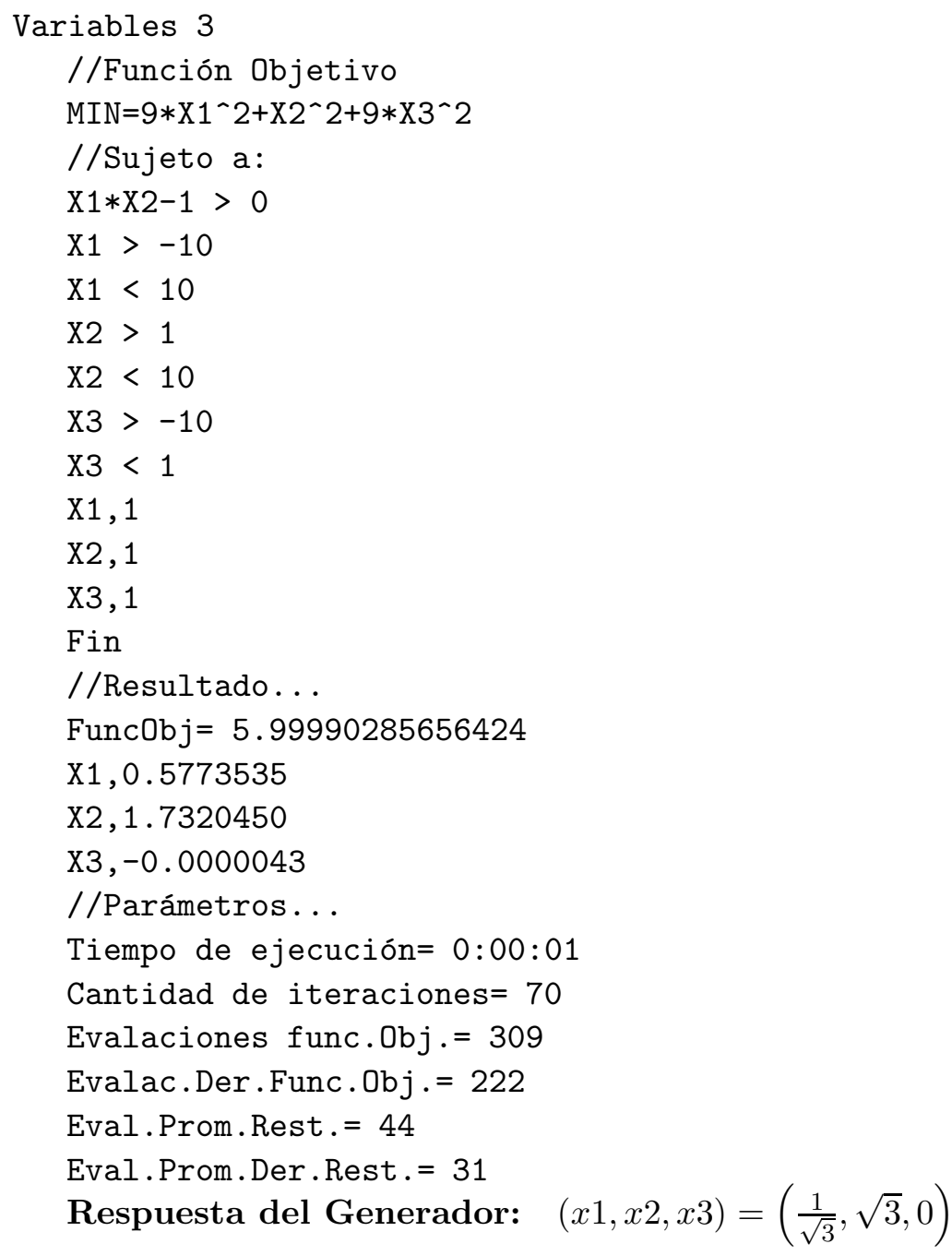

\section{Problema 39}

Variables 4

//Función Objetivo

MIN $=-X 1$

//Sujeto a:

$\mathrm{X} 2-\mathrm{X} 1 \wedge 3-\mathrm{X} 3{ }^{\wedge} 2=0$ 
$\mathrm{X} 1$ ^2-X2-X4^2=0
$\mathrm{X} 1,2$
$\mathrm{X} 2,2$
$\mathrm{X} 3,2$
$\mathrm{X} 4,2$
Fin
//Resultado...

FuncObj= -1.00007166675227

$\mathrm{X} 1,1.0000700$

$\mathrm{X} 2,1.0001800$

$\mathrm{X} 3,0.0000000$

$\mathrm{X} 4,0.0000000$

//Parámetros...

Tiempo de ejecución= 0:00:04

Cantidad de iteraciones $=203$

Evalaciones func. Obj. $=897$

Evalac.Der.Func. Obj.$=860$

Eval.Prom. Rest. $=454$

Eval.Prom.Der.Rest.$=430$

Respuesta del Generador: $\quad(\mathrm{X} 1, \mathrm{X} 2, \mathrm{X} 3, \mathrm{X} 4)=(1,1,0,0)$

\section{Problema 44}

Variables 4

//Función Objetivo

$\mathrm{MIN}=\mathrm{X} 1-\mathrm{X} 2-\mathrm{X} 3-\mathrm{X} 1 * \mathrm{X} 3+\mathrm{X} 1 * \mathrm{X} 4+\mathrm{X} 2 * \mathrm{X} 3-\mathrm{X} 2 * \mathrm{X} 4$

$/ /$ Sujeto a:

$8-\mathrm{X} 1-2 * \mathrm{X} 2>0$

$12-4 * \mathrm{X} 1-\mathrm{X} 2>0$

$12-3 * \mathrm{X} 1-4 * \mathrm{X} 2>0$

$8-2 * \mathrm{X} 3-\mathrm{X} 4>0$

$8-\mathrm{X} 3-2 * \mathrm{X} 4>0$

$5-\mathrm{X} 3-\mathrm{X} 4>0$

NO NEG $(1,4)$

Fin

//Resultado...

FuncObj $=-15.00071153231709$

$\mathrm{X} 1,-0.0000876$

$\mathrm{X} 2,3.0000680$

$\mathrm{X} 3,-0.0000728$

$\mathrm{X} 4,4.0000490$

//Parámetros...

Tiempo de ejecución= 0:00:05

Cantidad de iteraciones $=250$ 
Evalaciones func.Obj. $=1340$

Evalac. Der. Func. Obj.=1040

Eval. Prom. Rest. $=134$

Eval. Prom. Der. Rest. $=104$

Respuesta del Generador: $(\mathrm{X} 1, \mathrm{X} 2, \mathrm{X} 3, \mathrm{X} 4)=(0,3,0,4)$

\section{Problema 46}

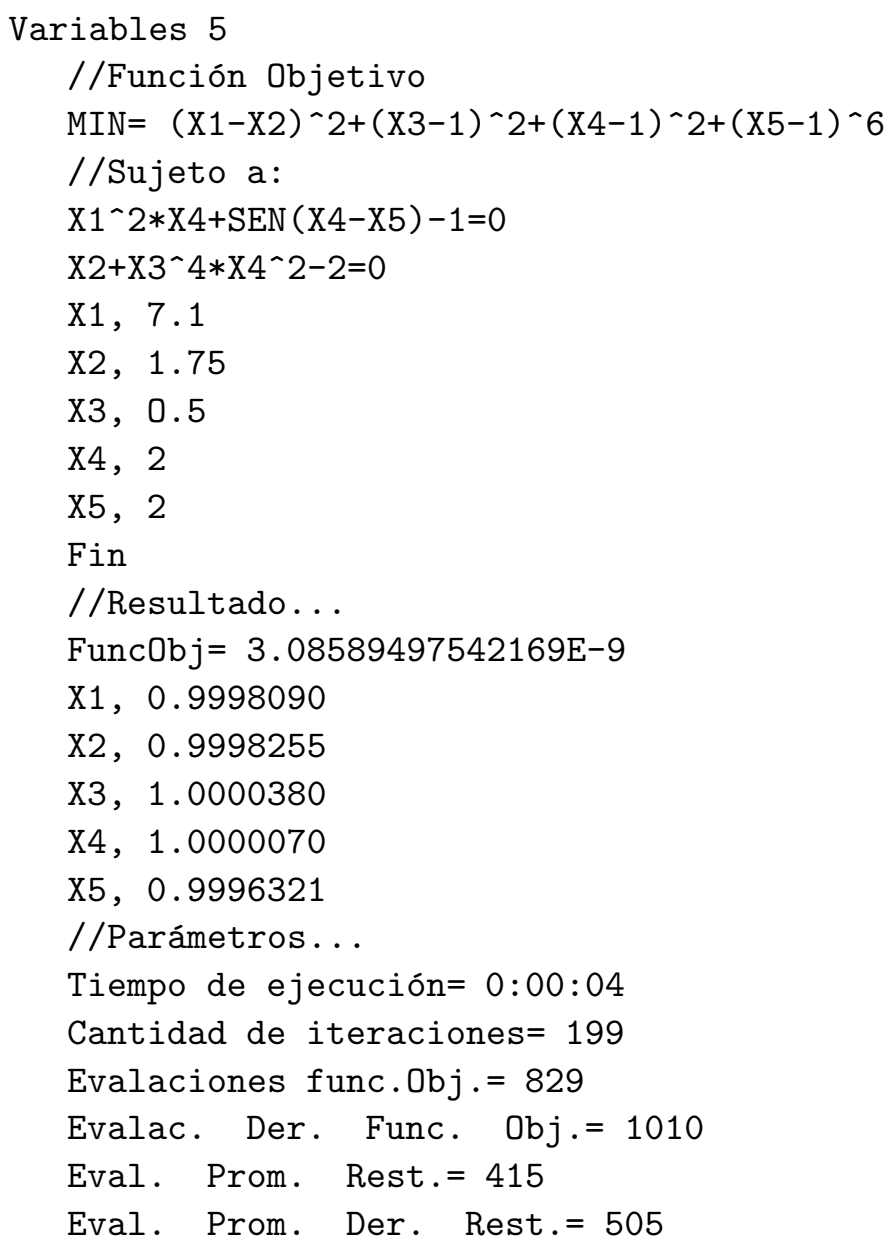

\section{Problema 60}

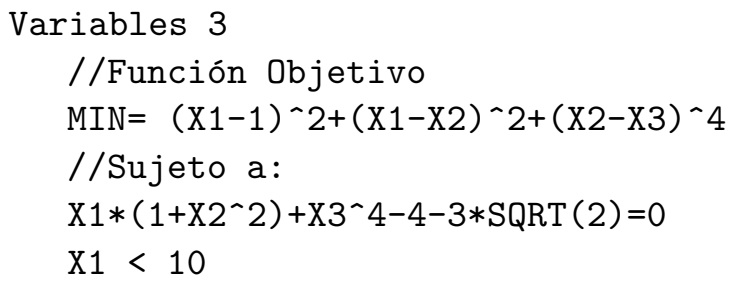




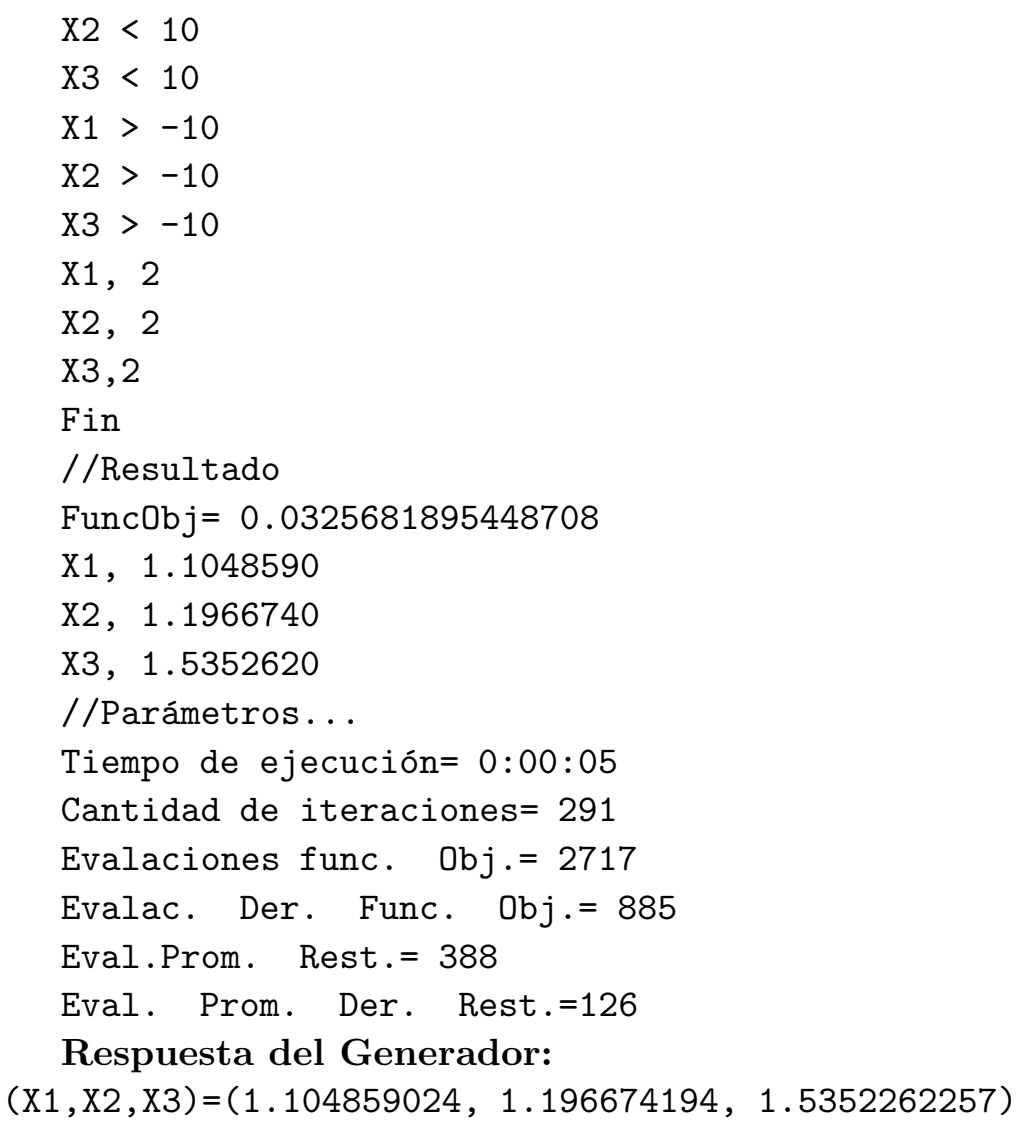

\section{Conclusiones}

OPTIMIZA ha sido validado con una clase amplia de funciones obteniéndose buenos resultados, superiores incluso a los de otros paquetes semejantes, con un $92 \%$ de efectividad.

En las estructuras de datos se utilizó un diseño Orientado a Objetos y la implementación de las clases se ajusta correctamente al problema planteado de forma que la aplicación es robusta y flexible.

La forma de intercambiar información entre el usuario y la máquina permite editar ficheros de forma natural; si se desea editarlo en el ambiente, este reduce la entrada de errores y le orienta al usuario cómo desarrollar su tarea.

Debido a la modularidad del diseño del sistema, la extensión de su programación y adición de nuevos algoritmos se hace sencilla. En particular, se trabaja en extender el alcance del sistema para resolver problemas no lineales en enteros.

\section{References}

[1] Luenberger, G. (1984) Programación Lineal y No Lineal. Addison-Wesley Iberoamericana, México. 
[2] Nocedal, J. (1992) "Theory of algorithms for unconstrained optimization", Acta Numerica 1: 199-222.

[3] Nocedal, J.; Liu Dong, C. (1989) "On the limited memory BFGS method for large-scale optimization", Mathematical Programming 45: 503-528.

[4] Hock, W.; Schittkokski, K. (1981) Test Examples for Nonlinear Programming Codes. Lecture Notes in Economics and Mathematical Systems 187, Springer, Berlin.

[5] Buckley; Lenir, A. (1983) "QN-like variable storage conjugate gradients", Mathematical Programming 27: 103-119.

[6] Nocedal, J. (1980) "Updating quasi-Newton matrices with limited storage", Mathematical Comput. 35: 773-782. 